\title{
Facing a disruptive threat: how can a nuclear medicine service be prepared for the coronavirus outbreak 2020?
}

\author{
Winnie Wing-Chuen Lam ${ }^{1,2} \cdot$ Kelvin Siu-Hoong Loke ${ }^{1,2} \cdot$ Wai Yin Wong ${ }^{1,2} \cdot$ David Chee-Eng Ng ${ }^{1,2}$
}

Received: 2 March 2020 / Accepted: 24 March 2020 / Published online: 30 March 2020

(C) Springer-Verlag GmbH Germany, part of Springer Nature 2020

\begin{abstract}
Purpose The aim of this short communication is to outline our experience in policies and processes of a nuclear medicine service during the COVID-19 outbreak in Singapore.

Methods We describe the key considerations of policies and processes that have been implemented in our nuclear medicine service since the first case of COVID-19 was confirmed in Singapore General Hospital on 23 January 2020, up to the present time.

Results Infection control, screening of patients and visitors, segregation of risk groups, segregation of staff and service continuity plans, communication and staff welfare, using electronic platforms for multi-disciplinary meetings and tele-reporting are discussed.

Conclusion Since our hospital received the first patient with COVID-19 in Singapore, our centre has managed 16 COVID-19 cases to date. There has not been any healthcare worker in our institution who has contracted COVID-19 through patient contact. We have highlighted for discussion some of the policies and processes to prepare a nuclear medicine service for the COVID-19 threat.
\end{abstract}

Keywords COVID-19 $\cdot$ SARS-CoV-2 $\cdot$ Nuclear medicine $\cdot$ Infection

\section{Introduction}

On 31 December 2019, China alerted the World Health Organization (WHO) to several cases of unusual pneumonia in the city of Wuhan, Hubei Province in central China $[1,2]$. On 7 January 2020, Chinese authorities identified the causative agent to be a new type of coronavirus. As the number of cases began to increase, in January 2020, China instituted the lock-down of several Hubei cities. The virus continued to spread to the other provinces of China, with the death toll in China at the end of January recorded as 170. Imported cases of

This article is part of the Topical Collection on Infection and inflammation

Winnie Wing-Chuen Lam

winnie.lam.w.c@singhealth.com.sg

1 Department of Nuclear Medicine and Molecular Imaging, Division of Radiological Sciences, Singapore General Hospital, Outram Road, Singapore 169608, Singapore

2 Radiological Sciences Academic Clinical Programme, Duke-NUS Medical School, 8 College Road, Singapore 169857, Singapore coronavirus infection were soon detected in several neighbouring countries such as South Korea, Japan, Thailand and Singapore within the same month. The first case of imported coronavirus in Singapore was identified on 23 January 2020. On 11 February 2020, the World Health Organization (WHO) announced the disease caused by the virus (SARS-CoV-2) would be called COVID-19. On 15 February 2020, a Chinese tourist died in France as a result of the virus, the first death in Europe. The WHO declared COVID-19 a pandemic on 11 March 2020. As of 14 March 2020, the global number of confirmed infected cases has exceeded 142,000, with more than 130 countries reporting cases, and the global reported death toll has risen to more than 5300 .

As of 15 March 2020, Singapore has seen 226 confirmed cases of COVID-19, with early detection and containment being currently the overall strategy to combat this disease, on a whole-of-government approach [3]. As for any medical institution, the key imperatives are to treat suspect and confirmed cases while paying attention to avoid intra-hospital and cross-institutional transmission between patients and staff. In this short communication sharing on our current experience in 
Singapore, the emphasis will be laid on the key considerations for a nuclear medicine service, particularly within an institutional/hospital setting, to prepare in terms of policies and processes, with the focused aim of avoiding intrainstitutional and cross-institutional spread of infection. Many of these policies and processes are common to the institution and radiological services.

\section{Key considerations of policies and processes}

\section{Infection control}

Key to avoiding transmission is adoption of good personal and institutional infection control practices appropriate to the overall situation, as well as different areas of workplace and different levels of risks among the patients.

Proper degrees of masking (surgical mask, N95 mask, eye protection) and other personal protective equipment (gowns, gloves) were quickly identified for doctors and staff handling different risk groups of patients and different categories of procedures. As evidence suggests that the SARS-CoV-2 virus was spread via droplets, procedures that involved patient coughing or otherwise producing droplets or aerosols (such as bronchoscopy or intubation) were considered higher risk procedures. For nuclear medicine practice, we temporarily halted the ventilation portion of the ventilation/perfusion (V/Q) scan during this outbreak. Angiographic-guided procedures such as Y-90 microspheres therapy, as well as radioiodine therapy, peptide receptor radionuclide therapy (PRRT) and Lutetium-177 PSMA therapy were deemed relatively aerosol-free and allowed to continue.

We kept to regular monitoring and even audits of adherence to infection control practices within the department during the heightened state of the outbreak. There was a need to provide staff with proper training on the use of surgical masks, or N95 masks, and various personal protective equipment.

\section{Screening of patients and visitors}

Screening of patients and visitors as they arrive in the department is crucial. Typical screening methodology includes recording relevant personal particulars, taking a targeted history of travel or history of recent close contacts with known clusters of disease, as well as any flu-like respiratory symptoms.

Temperature-taking at entrances acts as a guard, although it is also known that not all infected patients are symptomatic nor febrile. An isolation room had to be designated to temporarily hold any suspect cases of the infection who may have arrived at the department inadvertently. The purpose of this isolation room was to provide a safe area away from other patients, where any suspect cases can be reviewed by a physician for further management.
There are designated wards for COVID-19 isolation, acute respiratory infection (ARI) and general inpatients. Isolation or ARI cases expose staff to the risk of cross infection, so requests for such patients need to be vetted by the nuclear medicine physician for appropriateness.

\section{Segregation of risk groups}

Segregation of high-risk vs low-risk patients in terms of suspicion of COVID-19 was deemed important. This is to reduce the chance of intra-institutional spread, as well as to facilitate or simplify contact-tracing.

Segregation can be done in terms of space, where different separated zones within the department were designated for different types of risk. Alternatively, or additionally, segregation of patients can be achieved in terms of time (temporal separation). This method is particularly useful in facilities that are constrained in terms of layout of the department.

Procedures that potentially can cause coughing or exhalation of aerosol such as exercise stress for myocardial perfusion imaging should be carefully planned. To minimise time spent in the department for inpatients, we use the fastest 1-day protocol with pharmacological stress if possible.

We managed to segregate outpatients from inpatients for PET/CT scans, doing the latter after all the outpatients have been completed for the day. For general nuclear medicine scans, one scanning room was designated for scanning inpatients. Waiting time in the department should be minimised as far as possible. The names of the technologist, nurse and patient aide attending to each patient were recorded, to facilitate contact tracing, should the need arise.

There have been publications on the chest $\mathrm{CT}$ findings in COVID-19 patients [4-6]. These findings have been useful. We instituted a new requirement for CT (as part of PET/CT or SPECT/CT) images to be screened before allowing patients to leave the department. If there are CT changes suspicious for COVID-19, such as ground-glass opacities in a peripheral distribution, the referring clinician would be informed to decide on further management.

A summary of the segregation of risk groups is given in Table 1.

\section{Segregation of staff and service continuity plans}

Staff can be infected and become a nidus for infection within the hospital, and this can potentially lead to a massive shutdown of the service if no contingency plans are afoot. As was practised during the time of severe acute respiratory syndrome (SARS) in 2003 [7], our staff were segregated into 2 or more teams, which worked and rested in alternate shifts of 2 or more weeks, so that in the event of any contact with an unexpected case, the affected staff can stand-down while the other team 
Table 1 Segregation of risk groups of patients

Aerosol generating procedures, e.g. ventilation Temporarily halted. Radionuclide perfusion only scan for urgent cases. Non-urgent requests postportion of $\mathrm{V} / \mathrm{Q}$ scan poned.

Exercise stress for myocardial perfusion imaging

Segregation of inpatients and outpatients

Converted to pharmacological stress if possible.

Where possible, one scanning room designated for inpatients.

If physical segregation not possible, temporal segregation (inpatients after all outpatients) done.

Hybrid imaging with CT component

CT images of lungs screened before allowing patients to leave the department. Referring clinician informed if suspicious CT changes detected.

takes over. The segregated teams apply to nuclear medicine physicians, technologists, nurses and health care support staff.

In certain situations, staff segregation is even more critical. For example, certain designated staff such as those manning portable X-ray machines and ultrasound machines for highrisk suspect cases are segregated from the rest.

Staff are also strongly encouraged to monitor their temperature twice daily as a surveillance for early sign of potential infection. As a precautionary measure, staff are advised to practice social distancing and seek medical attention promptly when unwell.

\section{Communication and staff welfare}

Communication and staff welfare are also important. The chain is as strong as its weakest link. Inadequate communication throughout the staff levels can lead to uneven infection control practices and deficiencies while performing procedures for suspect cases of infection. This is particularly critical during an acute escalation of the epidemic. We had regular planned sessions of updates for staff on the occasionally fast-changing routine instructions for the hospital or institution, and this appeared to provide a sense of calmness, while giving all staff ample opportunity to ask questions, and be prepared and empowered.

Caring for patients during an uncertain epidemic can be psychologically draining for staff especially if it is prolonged. Staff should have adequate rest and hydration during times where they can de-mask. Naturally, all logistics supply of various types of masks, gowns, eye protection and even powered air-purifying respirator devices must be adequate, and seen to be adequate. These should be more than available for all staff as required, and the inventory should be kept constantly updated.

\section{Using electronic platforms for multi-disciplinary meetings and tele-reporting}

Technology was brought into more prominence in this outbreak. In view of staff segregation and restriction of staff movement across different institutions, tele-reporting was used to ensure continuity of the nuclear medicine service across different sites. Additionally, having large gatherings of doctors and other staff for meetings were discouraged. As a result, electronic videoconferencing platforms, e.g. Webex or Zoom [8], were used increasingly during this outbreak to conduct meetings and tumour board discussions.

One major issue relating to staff segregation is the difficulty of continuing with staff and residency training of doctors, that have to be posted across different institutions. Research with human subjects was largely postponed, as much as ethically possible.

Acknowledgements We acknowledge the assistance provided by all staff of the Department of Nuclear Medicine and Molecular Imaging and Division of Radiological Sciences, Singapore General Hospital.

\section{Compliance with ethical standards}

Conflict of interest The authors declare that they have no conflict of interest.

Ethical approval This article does not contain any studies with human participants or animals performed by any of the authors.

\section{References}

1. Zhu N, Zhang D, Wang W, et al. A novel coronavirus from patients with pneumonia in China, 2019. N Engl J Med. 2020;382:727-33.

2. Huang C, Wang Y, Li X, et al. Clinical features of patients infected with 2019 novel coronavirus in Wuhan, China. Lancet. 2020;395: 497-506.

3. Wong JEL, Leo YS, Tan CC. COVID-19 in Singapore-current experience. Critical global issues that require attention and action. JAMA. 2020. https://doi.org/10.1001/jama.2020.2467.

4. Ai T, Yang Z, Hou H, et al. Correlation of chest CT and RT-PCR testing in coronavirus disease 2019 (COVID-19) in China: A report of 1014 cases. Radiology. 2020. https://doi.org/10.1148/radiol. 2020200642.

5. $\mathrm{Xu} X, \mathrm{Yu} \mathrm{C}, \mathrm{Qu}$ J, et al. Imaging and clinical features of patients with 2019 novel coronavirus SARS-CoV-2. Eur J Nucl Med Mol Imaging. 2020. https://doi.org/10.1007/s00259-020-04735-9.

6. Qin C, Liu F, Yen T, et al. ${ }^{18}$ F-FDG PET/CT findings of COVID-19: a series of four highly suspected cases. Eur J Nucl Med Mol Imaging. 2020. https://doi.org/10.1007/s00259-020-04734-w. 
7. Tsou IYY, Goh JSK, Kaw GJL, et al. Severe acute respiratory syndrome: management and reconfiguration of a radiology department in an infection disease situation. Radiology. 2003;229:21-6.

8. Eagle T, Fasciani M, Preset A. Gartner critical capabilities for meeting solutions. 2019. https://www.gartner.com/doc/reprints?id=1-
1OEPTVC6\&ct $=190820 \&$ st $=$ sb \&elqTrackId=0b5ee44a36c $64 \mathrm{a}$ ce9bffb16c2794704b\&elqaid=264\&elqat=2. Accessed 29 Feb 2020.

Publisher's note Springer Nature remains neutral with regard to jurisdictional claims in published maps and institutional affiliations. 